\title{
Taking psychological well-being to heart
}

\author{
An Pan PhD, Kathryn M. Rexrode MD MPH
}

See related research article by Hamer and colleagues on page 1461 and at www.cmaj.ca/lookup/doi/10.1503/cmaj.111719

$\mathrm{I}$ n a related $C M A J$ article, Hamer and colleagues ${ }^{1}$ report results from the Health Survey for England showing an association between baseline psychological distress and subsequent increased risk of death due to cerebrovascular disease. Their results are in concordance with findings from previous observational studies and highlight the connection between psychological and cardiovascular health. In this commentary, we briefly review the existing literature and discuss the interpretation and implication of their findings.

In their study, Hamer and colleagues ${ }^{1}$ found that psychological distress was significantly associated with an increased risk of death from cerebrovascular causes. Compared with those without psychological distress, individuals who scored 1-3 and 4 or greater on the 12-item General Health Questionnaire (GHQ-12) had a $20 \%$ and $66 \%$ increased risk of death related to cerebrovascular disease, respectively, after adjustment for other risk factors. Similar associations were found for deaths from ischemic heart disease and other cardiovascular-related deaths.

The study by Hamer and colleagues ${ }^{1}$ adds to the existing literature and is consistent with prior studies linking psychological stress with cardiovascular disease, particularly stroke. For example, in the Caerphilly Study, ${ }^{2}$ significant psychological distress, as measured by the GHQ-30, was related to an increased risk of fatal ischemic stroke in men (relative risk $3.36,95 \%$ confidence interval 1.29-8.71). In the European Prospective Investigation into Cancer - Norfolk study, ${ }^{3}$ greater psychological distress (measured by a decrease of 1 standard deviation in score on the 5-item Mental Health Inventory) was associated with an $11 \%$ increased risk of stroke. In the Scottish Health Survey, Hamer and colleagues ${ }^{4}$ showed that psychological distress, as measured by the GHQ-12, was linked with an increased risk of total cardiovascular disease; however, the association was largely explained by behavioural factors, such as smoking and level of physical activity. Additionally, several of the individual components of psychological distress have been investigated as potential risk factors for cardio- vascular or cerebrovascular diseases. For example, depression has been consistently shown to predict future risk of coronary heart disease and stroke, ${ }^{5}$ whereas other studies have indicated that anxiety and stress are tied to increased cardiovascular risk. $^{6}$

The current literature supports the view that psychological distress may be toxic to the cardiovascular system; however, before applying the findings to clinical practice and public campaign, we need to consider potential limitations and remaining questions. First, most of the studies assessed psychological distress only at baseline when study participants were enrolled, and repeated measures were not available during follow-up. Thus, we know nothing about whether change in psychological status might affect cardiovascular health. Unlike diabetes and other chronic diseases, psychological status may change frequently over time. Therefore, from the current literature, we do not know whether the risk of cardiovascular disease is related to the cumulative exposure to psychological distress over a long period or a relatively short time before the onset of a cardiovascular disease event, or whether a latency period exists such that an episode of psychological distress would influence risk of remote cardiovascular disease.

Second, psychological distress is a constellation of various negative mood symptoms, and we do not know whether the individual components contribute equally or differentially to the increased risk. Many of the individual components have been shown to predict risk of cardiovascular disease in the general population. Moreover, effective therapies are available in

\section{- KEY POINTS}

- Psychological distress is associated with increased risk of stroke and cardiovascular disease.

- The mechanisms relating stress to increased cardiovascular risk are unclear at the moment.

- The cardiovascular benefits of treating psychological stress remain unproven, but clinicians should be mindful of traditional cardiovascular risk factors in those who are stressed and refer them for support and treatment. 
clinical practice for certain components, such as depression and sleep problems. However, there is no standard procedure to screen and treat psychological distress in primary care settings. Whereas instruments measuring psychological distress (e.g., the GHQ-12) may provide information on overall mental health status, more detailed interviews are required to diagnosis depression and anxiety with high specificity before considering treatment.

Third, the mechanisms relating psychological distress to increased risk for cardiovascular disease are still unclear. The environmental challenges that may induce psychological distress are highly diverse, ranging from socioeconomic status, marital and family conflict, job insecurity and stress, racial and gender discrimination, and life-altering events like bereavement or the diagnosis of a major disease. Therefore, we need to understand the specific psychological stressors to provide individualized therapy. There has been mounting evidence of physiologic alterations related to depression and stress that may increase risk for cardiovascular disease, including chronic inflammation, sympathetic nervous system activation, dysregulation of the hypothalamicpituitary-adrenocortical axis, platelet hyperactivity and endothelial dysfunction. ${ }^{7}$ Meanwhile, adverse behaviours, such as smoking, physical inactivity, poor diet and lack of medication adherence, as well as cardiometabolic abnormalities (e.g., obesity, metabolic syndrome, diabetes and hypertension), have been frequently linked to depression and mental distress. Some studies have suggested that behavioural factors may explain a substantial proportion of the variance linking psychological distress and risk for cardiovascular disease, ${ }^{4,8}$ but more studies are still warranted to disentangle the complex pathways.

Lastly, although psychological distress has been associated with adverse outcomes, the benefits of treatment have not been proven. Studies have suggested that pharmacological treatment of depression following stroke or cardiac events can improve depressive symptoms and may enhance quality of life, ${ }^{9}$ but whether treatment reduces secondary events and mortality is controversial. Cognitive behavioural therapy may provide additional benefits in conjunction with standard treatment. ${ }^{10}$ In light of the findings by Hamer and colleagues, ${ }^{1}$ we need further studies to test whether treatment aimed at alleviating psychological distress (e.g., pharmacological medications, cognitive behavioural therapy and lifestyle management) will reduce cardiovascular risk. A well-designed clinical trial may answer the question, but it will require a study of large sample size and sufficient long-term follow-up to test change in outcomes related to cardiovascular disease.

A vast body of literature has shown the interplay between mental health and physical wellness, and the current study adds additional evidence highlighting their interdependence. However, research is still needed to understand the causal relations and underlying mechanisms resulting in these associations, as well as to test whether interventions reduce the associated risk. Based on the available evidence, we highly recommend that clinicians pay enhanced attention to traditional cardiovascular risk factors in people with psychological distress and existing psychological illness (e.g., major depressive disorder or generalized anxiety disorder). Being mindful of patients' psychological health, and referring those with psychological distress for appropriate support and treatment, should improve their emotional well-being and may have a beneficial impact on their cardiovascular health as well.

\section{References}

1. Hamer M, Kivimaki M, Stamatakis E, et al. Psychological distress as a risk factor for death from cerebrovascular disease. CMAJ 2012;184:1461-66.

2. May M, McCarron P, Stansfeld S, et al. Does psychological distress predict the risk of ischemic stroke and transient ischemic attack? The Caerphilly Study. Stroke 2002;33:7-12.

3. Surtees PG, Wainwright NW, Luben RN, et al. Psychological distress, major depressive disorder and risk of stroke. Neurology 2008;70:788-94.

4. Hamer M, Molloy GJ, Stamatakis E. Psychological distress as a risk factor for cardiovascular events: pathophysiological and behavioral mechanisms. J Am Coll Cardiol 2008;52:2156-62.

5. Pan A, Sun Q, Okereke O, et al. Depression and the risk of stroke morbidity and mortality: a systematic review and metaanalysis. JAMA 2011;306:1241-9.

6. Roest AM, Martens EJ, de Jonge P, et al. Anxiety and risk of incident coronary heart disease: a meta-analysis. J Am Coll Cardiol 2010;56:38-46.

7. Musselman DL, Evans DL, Nemeroff CB. The relationship of depression to cardiovascular disease: epidemiology, biology, and treatment. Arch Gen Psychiatry 1998;55:580-92.

8. Whooley MA, de Jonge P, Vittinghoff E, et al. Depressive symptoms, health behaviors, and risk of cardiovascular events in patients with coronary heart disease. JAMA 2008;300:2379-88

9. Taylor D, Meader N, Bird V, et al. pharmacology subgroup of the National Institute for Health and Clinical Excellence Guideline Development Group for Depression in Chronic Physical Health Problems. Pharmacological interventions for people with depression and chronic physical health problems: systematic review and meta-analyses of safety and efficacy. Br J Psychiatry 2011;198:179-88

10. Gulliksson M, Burell G, Vessby B, et al. Randomized controlled trial of cognitive behavioral therapy v. standard treatment to prevent recurrent cardiovascular events in patients with coronary heart disease: Secondary Prevention in Uppsala Primary Health Care project (SUPRIM). Arch Intern Med 2011;171:134-40.

Affiliations: From the Department of Nutrition (Pan), Harvard School of Public Health, Boston, Mass.; and the Division of Preventive Medicine (Rexrode), Department of Medicine, Brigham and Women's Hospital and Harvard Medical School, Boston, Mass.

Contributors: The authors contributed equally to the writing and revising of the manuscript and approved the final version submitted for publication. 